\title{
Medida da pressão arterial e a produção científica de enfermeiros brasileiros
}

\author{
SCIENTIFIC PRODUCTION OF BRAZILIAN NURSES ON BLOOD PRESSURE MEASUREMENT \\ MEDIDA DE LA PRESION ARTERIAL Y LA PRODUCCIÓN CIENTÍFICA DE LOS ENFERMEROS \\ BRASILEÑOS
}

\section{Edna Apparecida Moura Arcuri' ${ }^{1}$, Thelma Leite de Araújo² $^{2}$, Eugênia Veludo Veiga ${ }^{3}$, Sonia Maria Junqueira Vasconcellos de Oliveira ${ }^{4}$, José Luiz Tatagiba Lamas ${ }^{5}$ Jair Lício Ferreira Santos ${ }^{6}$}

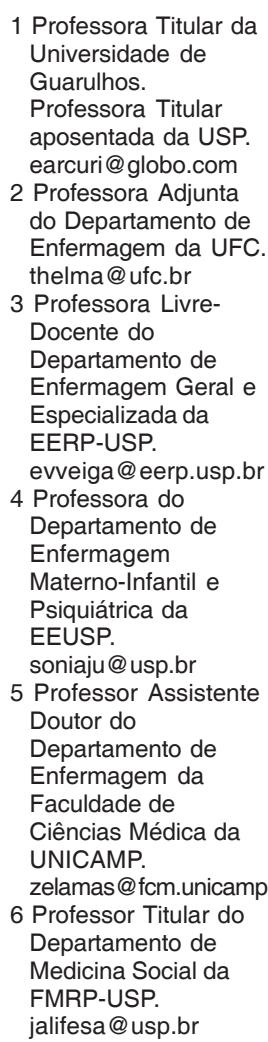

\begin{abstract}
RESUMO
No número anterior a este periódico, os autores apresentaram matéria comemorativa sobre a descoberta dos Sons de Korotkoff, em 1905. Relataram os determinantes históricos que resgatam o importante papel da Escola de Enfermagem da Universidade de São Paulo-EEUSP, no desenvolvimento da mais antiga linha de pesquisa de pressão arterial na enfermagem brasileira: "Influência da Largura do Manguito na Medida da Pressão Arterial". Neste artigo é apresentada a produção científica decorrente dos estudos realizados pelos doutores formados na EEUSP, enfatizando-se a trajetória de cada pesquisador em seus respectivos núcleos e laboratórios, com o objetivo de oferecer alguns indicadores que permitem avaliar, qualitativa-quantitativamente, $o$ impacto das pesquisas realizadas e a caracterização do conhecimento na área.
\end{abstract}

\section{DESCRITORES}

Pressão arterial.

Hipertensão.

Pesquisa em enfermagem.

\section{ABSTRACT}

In the previous issue of this journal the authors celebrated in an article the discovery of the Korotkoff sounds, in 1905, and presented the historical facts that determined the important role of the University of São Paulo's Nursing School (EEUSP) in the development of the oldest blood pressure research line in Brazilian Nursing: "Cuff Width Influence in Blood Pressure Measurement." This new article presents the scientific production derived from the studies carried out by nurses with a $\mathrm{PhD}$ from EEUSP during and after their doctoral programs in the area. The path each researcher followed in his/her group and laboratory is emphasized. The data presented is aimed at offering a number of qualitative and quantitative indicators that make possible to evaluate the impact of the research that they have carried out, as well as the development of knowledge in the area.

\section{KEY WORDS}

Blood pressure.

Hypertension.

Nursing research.

\section{RESUMEN}

En el número anterior de esta revista los autores celebraron el descubrimiento de los sonidos de Korotkoff en 1905 y relataron los determinantes históricos que rescatan el importante papel de la Escuela de Enfermería de la Universidad de São Paulo (EEUSP) en el desarrollo de la más antigua línea de investigación en la enfermería brasileña, "Influencia del Ancho del brazalete en la Medida de la Presión Arterial". En esta publicación se presenta la producción científica consecuente del trabajo realizado por los doctores formados en la EEUSP, enfatizándose la trayectoria de cada investigador en sus respectivos núcleos y laboratorios. Los datos presentados visan ofrecer algunos indicadores que permitan evaluar cualitativamente y cuantitativamente el impacto de las investigaciones realizadas y la caracterización del conocimiento en esfigmomanometría.

\section{DESCRIPTORES}

Presión arterial.

Hipertensión.

Investigación en enfermería. 


\section{INTRODUÇÃO}

Após um século da descoberta dos sons de Korotkoff, lacunas no conhecimento permanecem na área da medida da pressão, no diagnóstico precoce e tratamento da hipertensão e na avaliação dos fatores de risco para moléstias cardiovasculares. Raros são os centros de estudos em esfigmomanometria no exterior onde os pesquisadores deram continuidade às suas investigações, o que motivou os autores deste artigo, doutores na área da medida da pressão a relatar os trabalhos oriundos de seus núcleos de pesquisa, laboratórios e campos assistenciais, em diferentes unidades acadêmicas no país. No número anterior desse periódico foram relatados os estudos longitudinais realizados na tentativa de verificar as consequiências do uso do manguito de largura padrão no diagnóstico da hipertensão e resultantes complicações cardiovasculares. O critério da participação dos autores foi a formação como pesquisador na área da influência da largura do manguito na medida da pressão arterial, além do estatístico (Santos JLF) envolvido no desenvolvimento das cinco teses de doutorado e outras publicações de autoria dos referidos autores.

\section{Formação de pesquisadores e produção científica na Universidade Federal do Ceará}

Ao desenvolver na EEUSP, em 1994, a tese de doutorado "Conhecimento do enfermeiro na medida da pressão arterial", Araújo analisou, com grande cuidado, desde os aspectos anátomo-fisiológicos até a tecnologia de ponta na época, o que resultou na construção de um instrumento valioso para ensino de graduação, pós-graduação, programas de educação permanente e desenvolvimento de pesquisadores na área. A autora tinha sólida experiência no campo assistencial e no ensino da EEUSP, além de grande facilidade para analisar a literatura, o que contribuiu para o enriquecimento do instrumento de coleta de dados. Ressalta-se ainda, no referido estudo, a caracterização da falta de conhecimento sobre a influência da largura da medida, entre enfermeiros da área de cardiologia ${ }^{(1-3)}$.

Ao ingressar como docente no Programa de Pós-Graduação em Enfermagem da Universidade Federal do Ceará em 1996, definiu como área temática para seus estudos a esfigmomanometria e a assistência a portadores de alterações cardiovasculares, em especial a hipertensão arterial. Parte significativa dos alunos dos Cursos de Mestrado e Doutorado que atua ou vai atuar em Programas de Saúde da Família, atendendo, entre outros, o programa de tratamento da hipertensão arterial. Esta situação faz com que aspectos da assistência definam estudos de pós-graduação, que precisam ter forte vinculação com os problemas regionais e contribuir para a solução de problemas locais. Até o momento foram titulados quinze mestres e quatro doutores.

A maior parte dos trabalhos desenvolvidos está inserida na área temática, preocupando-se com a assistência prestada aos portadores de hipertensão arterial, enfocando em especial a adesão ao tratamento e a consulta de enfermagem ${ }^{(4)}$, a avaliação da pressão arterial em grupos de risco ${ }^{(5)}$ e o ensino da medida da pressão $\operatorname{arterial}^{(6)}$. Estes estudos contaram com auxílio de agências de fomento sob forma de bolsas e de auxílio financeiro e seus resultados estão divulgados em periódicos nacionais da enfermagem $^{(7-11)}$ e outros países, como Espanha ${ }^{(12)}$ e Cuba $^{(13)}$. Como produtos tecnológicos desenvolvidos, destacam-se um software para o ensino dos sinais vitais, produto decorrente de tese de doutorado e uma cartilha educativa para escolares com orientações sobre fatores de risco para alterações cardiovasculares.

O que mais chama atenção no trabalho realizado em Fortaleza é a expressiva interação docente assistencial que ocorre no desenvolvimento dos projetos, incluindo muitas vezes enfermeiros voluntários e alunos de diversos níveis de formação. Consequentemente, a autora e alguns colaboradores vêm ganhando espaço na literatura ibero-latina e portuguesa, com diversas publicações em periódicos internacionais, o que representa valiosa contribuição para o desenvolvimento dos pesquisadores da área, como também para a atualização dos enfermeiros na Europa e América Latina.

\section{A integração do ensino, assistência e pesquisa na Oficina de Projetos em Esfigmomanometria da Escola de Enfermagem de Ribeirão Preto}

Mesmo antes da formação do primeiro doutor na área da esfigmomanometria, foi grande o interesse do Departamento de Enfermagem Médica e Especializada da EERP na formação de pesquisadores na área de hipertensão. Já na década de 70 foi desenvolvido um estudo ${ }^{(14)}$, que demonstrou como a correta avaliação da pressão diferencial sistólica/ diastólica (pressão de pulso), associada às características do quarto e quinto sons de Korotkoff, constitui importante parâmetro na identificação de alterações relacionadas à estenose ou insuficiência aórtica, fundamental ao encaminhamento precoce do indivíduo que necessita ser investigado.

Em 1989, Veiga e alguns colegas propuseram um laboratório de pesquisa associada à assistência e ensino, denominado Oficina de Projetos em Esfigmomanometria (OPE), iniciando suas atividades com 6 bolsas em nível de iniciação científica: alunos do Programa Especial de Treinamento; com bolsa trabalho do COSEAS, e posteriormente alunos do PIBIC/CNPq, cujas temáticas envolviam detecção precoce de valores de pressão arterial elevados e a hipertensão como um dos mais importantes fatores de risco cardiovascular ${ }^{(15-17)}$. Ao final da década de 80 iniciou-se atividades de extensão na região (palestras, cursos de atualizações em Medida Indireta da Pressão Arterial, detecção precoce dos indivíduos de risco, entre outros) em conjunto com a Liga de Diagnóstico, Tratamento e Controle da Hipertensão Arterial de Ribeirão Preto.

O grupo da OPE teve também participação efetiva na criação, coordenação e implementação do Programa de Aprimoramento Multiprofissional de Hipertensão Arterial na Oficina de Projetos em Esfigmomanometria na EERP, con- 
tribuindo para a atualização de outras pessoas interessadas no tema, fortalecendo as bases científicas na atenção ao hipertenso, no âmbito da pesquisa e do ensino em esfigmomanometria. Outros docentes ${ }^{(18)}$ tiveram forte participação nos projetos iniciais da oficina e posteriormente outras investigações foram conduzidas, formando um grupo com expressiva produção na área da hipertensão. $\mathrm{O}$ trabalho realizado em Ribeirão Preto merece uma análise própria, pois apenas alguns estudos encontram-se citados nesta publicação ${ }^{(15-21)}$, incluindo pesquisas com ácido nítrico.

Um fato inédito a ser destacado é a iniciativa de Veiga em questionar, em sua tese de Livre Docência, os aspectos éticos concernentes ao procedimento de medida da pressão $\operatorname{arterial}^{(22)}$. A autora atesta que é da responsabilidade das instituições prover equipamentos em número e em condições adequadas, que garantam a obtenção de valores precisos, com atualização e educação permanente dos profissionais que atuam nessa área. Além disso, afirma que:

Urge a organização de esforços que transformem a prática assistencial, em respeito aos preceitos éticos e legais, para que não venham ocorrer possíveis danos, relacionados à imprudência, negligência e imperícia ${ }^{(22)}$.

\section{A formação de pesquisadores na EEUSP na área da medida da pressão arterial em gestantes}

O prejuízo que o manguito de largura padrão pode acarretar à avaliação da pressão arterial no período gestacional foi constatado por Oliveira, que a partir da formação no curso de doutorado, na área da esfigmomanometria, iniciou a orientação dos alunos de iniciação científica e do curso de mestrado. Os dados referentes à tese de doutorado desenvolvida pela autora foram publicados em revista internacional A (Journal of Advanced Nursing) ${ }^{(23)}$, além de outras publicações sobre a medida ${ }^{(24)}$. A publicação no periódico editado pela Blackwell Science foi avaliada entre centenas de artigos publicados nessa revista durante sete anos, tendo sido classificado entre aqueles (37\%) cujo cálculo da amostra atendia o rigor estatístico esperado para uma revista de alto impacto ${ }^{(25)}$, como nos demais cálculos feitos nos estudos aqui citados.

Nos últimos cinco anos as investigações de Oliveira vêm sendo direcionadas na área da medida da pressão arterial na gestante $^{(26-27)}$, hipertensão na gravidez ${ }^{(28-29)}$, eclampsia e complicações na gravidez ${ }^{(30)}$. Os dados desses estudos foram divulgados em dez eventos internacionais e nove nacionais. Alguns estudos envolvem a participação de outros pesquisadores da área de obstetrícia, docentes da EEUSP, os quais unindo esforços vêm fortalecendo o desenvolvimento de pesquisas interdisciplinares nas áreas em apreço, assim como as publicações dos resultados em revistas nacionais e internacionais de impacto.

Com sólida formação na área de obstetrícia e doutorado em esfigmomanometria, Oliveira orientou seis projetos do
PIBIC/CNPq, três trabalhos de conclusão de curso de graduação e um mestrado, entre outros em andamento. Os esforços na divulgação desses estudos em eventos nacionais e no exterior vêm contribuindo para focar a atenção de alunos e profissionais da área obstétrica para avaliar com maior precisão a pressão arterial no período gestacional, condição imprescindível à detecção precoce de anormalidades.

\section{A formação de pesquisadores e os estudos realizados na UNICAMP}

Durante o desenvolvimento da tese de doutorado de Lamas, docente da Universidade Estadual de Campinas (UNICAMP) que teve formação na EEUSP, dois assuntos polêmicos foram estudados: a comparação de registros intrarteriais com registros diretos de pressão arterial em função do manguito de largura correta na artéria braquial e o uso da campânula do estetoscópio infantil ou o diafragma para a melhor ausculta dos sons de korotkoff, que resultaram em publicações ${ }^{(31-32)}$.

Centenas de pesquisas realizadas durante o século XX tentaram comparar os registros diretos da pressão na artéria com os obtidos pelo método auscultatório ou oscilométrico, porém o estudo de Lamas foi o único que controlou o erro provocado pela largura inadequada do manguito, utilizando braçadeiras com largura correspondente a $40 \%$ da circunferência do braço. A comparação entre os registros intrarteriais com os indiretos confirmou que as diferenças com o manguito padrão foram maiores do que aquelas constatadas com o manguito correto, prejudicando principalmente a avaliação da pressão sistólica.

A partir de sua formação Lamas passou a investigar temas ligados à prática rotineira da medida da pressão arterial por profissionais de enfermagem, como os erros cometidos durante o procedimento de medida ${ }^{(33-34)}$. Sua produção começou a ser divulgada em congressos nacionais e internacionais, incluindo os resultados de diversos projetos de iniciação com bolsas da FAPESP e CNPq ${ }^{(35-37)}$. Destaca-se também o estudo realizado para validar a tabela de correção de valores de pressão arterial proposta por Maxwell em 1982, cujos resultados confirmaram a inadequação do instrumento, em função de grandes variações interindividuais observadas ${ }^{(38-40)}$, em consonância com as observações de pesquisadora brasileira ${ }^{(41)}$. A produção do autor incluiu orientações em parceria com pesquisadores da área de sono e ritmos biológicos, que resultou na apresentação de trabalhos em eventos internacionais ${ }^{(42-43)}$. Aumentam cada vez mais os alunos com pesquisa esfigmomanométrica na UNICAMP, diversos com bolsas da FAPESP, totalizando em poucos anos 12 acadêmicos.

\section{Impacto da linha de pesquisa e a contribuição na caracterização do conhecimento}

Análise quantitativa - indica a formação de dez doutores na área, mais de vinte mestres e dezenas de orientações de iniciação científica. Arcuri defendeu sua tese em 1985, portanto 
sua produção é de 20 anos e dos demais autores deste artigo é de 11, 10, 8 e 5 anos. Apesar da grande quantidade de dados publicados no Brasil e exterior, a produção quantitativa nos últimos anos foi prejudicada pelo hiato na produção do pesquisador mais antigo, em razão da priorização aos estudos longitudinais na área. Nos últimos cinco anos dedicou-se à finalização do estudo iniciado em 1983, acreditando que a confirmação das hipóteses iniciais seria fundamental para o avanço do conhecimento, priorizando o acompanhamento das 492 pessoas da amostra ao longo de 20 anos, todos reavaliados em 2003.

No entanto, o desafio de pesquisar com diversas larguras de manguito não foi ainda vencido, pois os instrumentos modernos como os usados na monitoração ambulatorial da pressão arterial (MAPA), fundamentam-se nos mesmos princípios dos esfigmomanômetros tradicionais, o que não evita o erro causado pelas dimensões do manguito.

Cabe ressaltar que no Brasil, um dos principais assessores da FAPESP na área da hipertensão até anos recentes, sempre considerou que só existia hiperestimação da pressão por manguitos inadequados, e tentou desestimular a pesquisa desde 1985 , na primeira apresentação dos dados referentes à tese de doutorado. O parecer negando auxílio em 1991 afirmava que o assunto estava resolvido há 40 anos, teor idêntico ao parecer da enfermeira da American Heart Association que assessorava a revista Nursing Research em 1987, o que revelava a falta de conhecimento, nas duas instituições, de assessores com formação específica para julgar a matéria. Nos anos recentes, contudo, as hipóteses começam a ser citadas por sociedades e estudos multicêntricos de fatores de risco ${ }^{(44)}$. Entretanto, foi durante o programa de pós-doutorado realizado com o apoio da FAPESP, nas Universidades de Oxford e Milão, graças aos esforços do Prof. Goldemberg, reitor da USP na ocasião, que se deu início à discussão e divulgação dos dados brasileiros, durante os lectures apresentados em 1986, a convite das duas universidades.

Apesar da extensa produção de alguns doutores formados, os fatos relatados contribuíram inicialmente para dificultar a publicação dos primeiros resultados em revistas com impacto na área, que foram escolhidas devido ao caráter internacionalmente inédito de muitos protocolos. Contudo, os autores vêm enriquecendo o acervo nacional e internacional e consideram a produção quantitativamente satisfatória, pois apenas uma parte da produção encontra-se referenciada nesta publicação. Merece destaque a extensa produção de Araújo, fruto do trabalho que vem desenvolvendo na Universidade Federal do Ceará.

Análise qualitativa - a despeito de terem sido poucas as publicações na íntegra dos achados iniciais na década de 80 , dada à dificuldade de ter sido aceito que a hipoestimação da pressão era um fenômeno que existia como a hiperestimação, os fatos que permitem ao leitor avaliar os aspectos qualitativos foram os seguintes:

1986 - Aceitação do pesquisador mais antigo (Arcuri) para realizar programa de pós-doutorado nas tradicionais Universidades de Oxford e Milão. A qualidade do estudo (dados inéditos) resultou em convite para apresentação de lecture na Universidade de Oxford, com a presença do presidente da British Hypertension Society na ocasião, e na Universidade de Milão. Outra indicação qualitativa foi a discussão dos dados na Escola de Saúde Pública de Londres, com o Professor Rose, epidemiologista com grande conhecimento na área, o único que abordou a falta de resposta das pessoas magras ao tratamento anti-hipertensivo. Além dos lectures mencionados, os dados foram discutidos com a assessora de hipertensão da OMS, em Geneve.

1987 - Carta do Prof. Morais, Reitor da USP na ocasião, cumprimentando pela repercussão dos estudos e contribuição para a área de esfigmomanometria.

1988 - Dados demonstrando que a pressão diferencial (pressão de pulso) é influenciada pela largura do manguito são publicados em revista internacional $\mathrm{A}^{(45)}$.

1989 - Congresso Europeu de Hipertensão: Hipóteses levantadas para explicar a maior mortalidade no magro hipertenso receberam destaque na revista Controversies in Cardiology ${ }^{(46)}$, que embora não indexada e patrocinada pelo Laboratório Zambom, seu corpo editorial era formado pelo professor Alberto Zanchetti, presidente do congresso. Foi enfatizado que a hipoestimação nos registros (falta de diagnóstico) era mais importante que a hiperestimação. As hipóteses foram publicadas no Journal of Hypertension, periódico de forte impacto na área ${ }^{(47)}$.

1989 - A convite do Prof. Décourt, foi publicado nos Arquivos Brasileiros de Cardiologia, como Conferência, o tema "Manguito do Esfigmomanômetro e Diagnóstico da Hipertensão”(48).

1992 - Enfermeira da American Heart Association, que mais tarde foi sua presidente, solicitou consultoria referente à matéria publicada de 1993, uma das revisões das recomendações da AHA para medida de pressão.

1996 - Os achados dos pesquisadores aqui citados, foram mencionados por $\mathrm{O}^{\prime}$ brien no encerramento da comemoração do centenário do esfigmomanômetro de Riva Rocci, Dublin, Irlanda. O enfoque era referente aos desafios da medida correta no século XXI.

2004 - Dados de 20 anos de seguimento de 492 pessoas são aceitos para apresentação no Congresso da Sociedade 
Internacional de Hipertensão. Chamou atenção o fato de que entre os sujeitos que apresentaram níveis elevados de pressão em 1983 (grupo de risco) apenas quando utilizado o manguito de largura correta, 57\% são hoje hipertensos. Entre os que apresentavam níveis normais de pressão com o manguito correto ou padrão naquela ocasião, a ocorrência atingiu $26 \%$. Merece comentar que entre as 48 mortes observadas, 37 ocorreram no grupo de risco ${ }^{(49)}$, o que merece outras investigações.

2005 - No congresso da Sociedade Interamericana de Hipertensão realizado em Cancun, México, foi demonstrado que após 20 anos as complicações cardiovasculares apresentavam-se inversamente proporcionais à largura do manguito utilizado, comprovando a hipótese de prejuízo no diagnóstico precoce da hipertensão ${ }^{(46)}$. Sinais e sintomas como taquicardia, cefaléia, tonturas e, cansaço, além de infarto, AVC, eclampsia e préeclampsia, descolamento prematuro da placenta, foram complicações observadas com certa frequiência no estudo longitudinal $^{(50)}$. Diante de tal realidade, os autores criticaram e desafiaram as decisões da American Heart Association de 2005, que continuam referindo a razão circunferência braquial/largura de manguito de 0.40 , porém estipularam as larguras de 12 e $16 \mathrm{~cm}$ para circunferência braquiais com 22 a $26 \mathrm{~cm}$ e 27 a $52 \mathrm{~cm}$, respectivamente. Decisões baseadas em soluções práticas, visando apenas o preocupante número de obesos (sobretudo nos EEUU), aos quais também não é oferecida a solução correta, agrava o problema do alto custo do diagnóstico e tratamento das complicações da hipertensão. Ressalta-se, entretanto, que nossas críticas residem apenas no que concerne às dimensões do manguito, pois a revisão de 2005 é a mais completa já apresentada, reconhecendo-se nela os esforços e o valioso papel educativo do grupo de trabalho da Associação Norte-americana.

2005 - Os estudos ${ }^{(45,47)}$ publicados em periódicos de impacto e citados no JCR (Journal of Citation Reports) foram incluídos no Guidelines para a medida da pressão da American Heart Association/2005.

\section{CONSIDERAÇÕES FINAIS}

Após um século de esfigmomanometria, diversos problemas continuam sem solução. O reconhecimento internacional decorrente de publicações (qualis CAPES IA, B e C) e citações em periódicos de impacto, vem despertando interesse de intercâmbio por colegas do exterior (Canadá, Europa, EEUU, México). Talvez um esforço internacional da área da enfermagem possa representar uma esperança para a solução de um dos maiores desafios no campo do diagnóstico correto da hipertensão.

Com respeito à caracterização do conhecimento na área, os autores consideram que as pesquisas que realizaram nos últimos 20 anos evidenciaram:
- A hipoestimação da pressão arterial é um fenômeno que ocorre como a hiperestimação, porém suas conseqüências na falta de diagnóstico e tratamento da hipertensão arterial a tornam um grave problema.

- As mulheres não têm pressão arterial fisiologicamente baixa; trata-se de hipoestimação dos níveis registrados pelo manguito de largura padrão, embora a pressão arterial no sexo feminino seja um pouco menor que no masculino, antes dos 45 anos (fisiologicamente).

- A correlação inversa entre o índice de massa corpórea e mortalidade em hipertensos, e a falta de resposta ao tratamento nas pessoas magras, conforme demonstrado por vários autores, é decorrente da falta de diagnóstico precoce e tratamento adequado, devido à hipoestimação pelo manguito padrão em braços com circunferência menor que $29 \mathrm{~cm}$, sobretudo naqueles finos entre 20 e $25 \mathrm{~cm}$.

- A largura do manguito determina alterações na pressão de pulso (pressão diferencial sistólica/diastólica), que pode ser diminuída em pessoas magras e aumentada nas obesas.

- O uso de manguitos corretos evita erros de diagnóstico de pré-eclampsia no período gestacional.

- A avaliação pediátrica é igualmente prejudicada por falta de manguitos infantis, impedindo o diagnóstico precoce e tratamento também nas crianças obesas ou magras.

- A campânula infantil propicia melhores condições de ausculta na medida da pressão no antebraço.

- O conhecimento de muitos profissionais sobre a medida da pressão é deficitário, o procedimento é executado sem bases científicas e inexistem programas de educação permanente.

- A análise de qualquer variável relacionada à medida da pressão arterial, como dos fatores de risco para moléstias cardiovasculares, drogas anti-hipertensivas, ação de substâncias e drogas vaso-ativas, variáveis demográficas, curva de distribuição de freqüência das pressões sistólicas e diastólicas, etc, é prejudicada pela influência do manguito padrão na medida da pressão arterial. Tal fato é responsável por tanta polêmica detectada na literatura.

Outro indicador é que grande parte dos estudos desenvolvidos e das inúmeras comunicações em eventos científicos recebeu diferentes modalidades de fomento em nível de iniciação e pós-graduação, stricto e lato senso, da FAPESP e CNPq (bolsas de PIBIC, aperfeiçoamento, apoio técnico, mestrado e doutorado). 


\section{REFERÊNCIAS}

1. Araújo TL. Medida indireta da pressão arterial: aspectos conceituais e caracterização do conhecimento do enfermeiro [tese]. São Paulo: Escola de Enfermagem, Universidade de São Paulo; 1994.

2. Araujo TL, Arcuri EAM. Medida indireta da pressão arterial: aspectos conceituais e caracterização do conhecimento do enfermeiro. Rev Lat Am Enferm. 1998;6(4):21-9.

3. Araújo TL, Arcuri EAM, Martins E. Instrumentação na medida da pressão arterial: aspectos históricos, conceituais e fontes de erro. Rev Esc Enferm USP. 1998;32(1):33-41.

4. Moreira TMM. Tecnologia de cuidado na busca da adesão ao tratamento da hipertensão arterial: desenvolvimento e avaliação de uma experiência em Fortaleza-CE [dissertação]. Fortaleza: Universidade Federal do Ceará; 2003.

5. Chaves ES. Avaliação da pressão arterial em crianças e adolescentes com história familiar de hipertensão arterial [dissertação]. Fortaleza: Universidade Federal do Ceará; 2004.

6. Lopes MVO. Validação de software educativo para auxílio ao ensino de sinais vitais [tese]. Fortaleza: Universidade Federal do Ceará; 2001

7. Oliveira TC, Araújo TL, Melo EM, Almeida DT. Avaliação do processo adaptativo de um idoso portador de hipertensão arterial. Rev Lat Am Enferm. 2002;10(4):530-43.

8. Oliveira TC, Araújo TL. Mecanismos desenvolvidos por idosos para enfrentar a hipertensão arterial. Rev Esc Enferm USP. 2002;36(3):276-81.

9. Maciel ICF, Araújo TL. Consulta de enfermagem: análise das ações junto a programas de hipertensão arterial, em Fortaleza. Rev Lat Am Enferm. 2003;11(2):207-14.

10. Lopes MVO, Araújo TL. Avaliação de alunos e professores acerca do software sinais vitais. Rev Esc Enferm USP. 2004;38(4):438-47.

11. Guedes NG, Costa FBC, Moreira RP, Cavalcante TF, Chaves ES, Araújo TL. Crises hipertensivas em portadores de hipertensão arterial em tratamento ambulatorial. Rev Esc Enferm USP. 2005;39(2):181-9.

12. Moreira TMM, Araújo TL, Chaves ES. La investigación perfeccionando la práctica de enfermería: técnica del cuidado para la adhesión al tratamiento de la hipertensión arterial. Enferm Integral. 2004;66(1):4-9.

13. Silva VM, Lopes MVO, Araújo TL. Validación de un recurso lúdico para la educación en salud cardiovascular. Rev Cubana Enferm. 2004;20(3):1-6.

14. Vinha VP. Estudo da pressão arterial em policiais militares do grupo etário 20-50 anos [tese]. Ribeirão Preto: Escola de Enfermagem de Ribeirão Preto, Universidade de São Paulo; 1972.

15. Veiga EV, Nogueira MS, Cárnio EC, Marques S, Lavrador MAS, Nobre F. Avaliação de técnicas da medida da pressão arterial pelos profissionais de saúde. Arq Bras Cardiol. 2003; 80(1):83-9.
16. Veiga EV, Robazzi MLCC, Nogueira MS, Takamura MS, Hayashida M. Estudos dos fatores de risco da hipertensão arterial: conhecimento e exposição. Rev Soc Cardiol Estado São Paulo 1993;3(6):1-5.

17. Veiga EV, Arcuri EAM, Santos JLF. Indirect blood pressure measurement as a function of cuff width in 6 to 10 years old school children [text on the Internet]. Indianapolis: Sigma Theta Tau International; 1998 [cited 1998 May 15]. Available from: http://www.stti.iupui.edu/rnr/register/

18. Robazzi MLCC, Veiga EV, Nogueira MS, Hayashida M, CaronRufino M. Valores da pressão arterial em trabalhadores de uma instituição universitária. Ciênc Enferm Rev Ibero Am Invest. 2002;8(1):57-65.

19. Alves LMM, Nogueira MS, Veiga EV, Godoy S, Cárnio EC. White coat hypertension and nursing care. Can J Cardiovasc Nurs. 2003;13(3):29-34.

20. Santiago CM, Fernandes CH, Nogueira MS, Veiga EV, Cárnio EC. Utilização do óxido nítrico como terapêutica: implicações para a enfermagem. Rev Lat Am Enferm. 2000;8(6):76-82.

21. Rodrigues RAP, Marques S, Mendes RT, Oliveira CCL, Mendes MMR, Veiga EV. Adesão ao tratamento da idosa hipertensa. Arq Bras Med. 1996;70(9):451-6.

22. Veiga EV. Esfigmomanometria indireta e a prática clínica: reflexões e perspectivas [tese]. Ribeirão Preto: Escola de Enfermagem, Universidade de São Paulo; 2003.

23. Oliveira SMJV, Arcuri EAM, Santos JLF. Cuff width influence on blood pressure measurement during pregnant-puerperal cycle. J Adv Nurs. 2002;38(2):180-9.

24. Oliveira SMJV, Arcuri EAM. Medida da pressão em gestantes. Rev Lat Am Enferm. 1997;5(3):49-55.

25. Williamson GR. Misrepresenting random sampling? A systematic review of research papers in the Journal of Advanced Nursing. J Adv Nurs. 2003;44(3):278-88.

26. Oliveira SMJV, Lima APF. Medida indireta da pressão arterial em gestantes: parâmetros utilizados pela equipe de enfermagem. Acta Paul Enferm. 2002;15(3):27-34.

27. Oliveira SMJV. Medida de pressão arterial na gestante. Rev Bras Hipertens. 2000;7(1):59-64.

28. Oliveira SMJV, Persinotto MOA. Revisão de literatura em enfermagem sobre hipertensão arterial na gravidez. Rev Esc Enferm USP. 2001;35(3):214-22.

29. Oliveira SMJV, Freitas P. Gestantes com hipertensão arterial: perfil e conduta de enfermagem. Rev Baiana Enferm. 2002;17(3):23-33

30.Oliveira SMJV, Domingues CA. Prevalência de eclampsia em parturientes. Rev Ginecol Obstet. 2004;15(3):148-54.

31. Lamas JLT, Arcuri EAM. Cuál es la mejor pieza de auscultación para medir la presión arterial? Temas Enferm Actual. 2002;10(48):12-6. 
32. Lamas JLT, Arcuri EAM, Brito CM, Cruz KCT. Blood pressure measurement: comparison between direct and indirect values as a function of cuff width. In: Programme of the Annual Nursing Research Conference; 2000 Apr. 13-15; Sheffield, England. Sheffield: Royal College of Nursing of the United Kindon Research Society. 2000. p. 41.

33. Lamas JLT, Arcuri EAM, Brito CM, Cruz KCT. Medida direta da pressão arterial versus medida indireta em função da largura do manguito. In: Anais do $11^{\circ}$ Seminário Nacional de Pesquisa em Enfermagem; 2001 maio 27-30; Belém [CD-ROM]. Belém: ABEn-Seção PA; 2001.

34. Berno CBF, Takeiti GM, Lamas LFT. Condutas de profissionais de enfermagem na medida da pressão arterial. In: Resumos do 53 Congresso Brasileiro de Enfermagem; 2001 out. 9-14; Curitiba [CD-ROM]. Curitiba: ABEn-Seção PR; 2001.

35. Lamas JLT, Spinella C. Detecção de fatores de risco para hipertensão arterial e níveis pressóricos de adolescentes trabalhadores. In: Libro de Resúmenes do $9^{\circ}$ Colóquio Panamericano de Investigación en Enfermeria; 2004 nov. 1-3; Lima [CD-ROM]. Washington: OPAS; 2004.

36. Lamas JLT, Berno CBF, Takeiti GM. Erros cometidos por profissionais de enfermagem na medida rotineira da pressão arterial. Rev Paul Enferm. 2003;22(2):141-8.

37. Lamas JLT, Benedito MO. Efeito agudo da atividade física sobre a pressão arterial. In: Livro de Resumos do $12^{\circ}$ Congresso de Iniciação Científica da UNICAMP; 2004 out. 22-23; Campinas. Campinas: UNICAMP; 2004. v. 1, p. 33.

38. Maxwell MH, Warks AB, Schroth PC, Karan M. Error in blood pressure measurement due to incorrect cuff size in obese patients. Lancet. 1982;2:33-6.

39. Pedroso MR, Lamas JLT. Adequação do uso de tabelas de correção de valores de pressão arterial em indivíduos obesos. In: Livro de Resumos do $12^{\circ}$ Congresso de Iniciação Científica da UNICAMP; 2004 out. 22-23; Campinas. Campinas: UNICAMP; 2004. v. 1, p. 33.

40. Lamas JLT, Pedroso MR, Salvatori AM. Correção de valores de pressão arterial obtidos com manguito de largura padrão: comparação a valores obtidos com manguito de largura correta. Rev Soc Cardiol Estado São Paulo. 2004;14(Supl):83.
41. Arcuri EAM, Rocha e Silva M. Correct cuff width versus standard cuff width: wide scattering imposes a limitation to the use of correction of errors in indirect blood pressure determinations. Braz J Med Biol Res. 1986;19(4/5):522a.

42. Rabelo JA, Ceolim MF, Lamas JLT. Sleep in hypertensive obese and non-obese subjects. In: Livro de Resumos do $1^{\circ}$ Simpósio Internacional Inovação e Difusão do Conhecimento em Enfermagem; 2003 ago. 5-7; Ribeirão Preto. Ribeirão Preto: EERP/USP; 2003. p. 221.

43. Ceolim MF, Lamas JLT, Ramos FP, Rabelo JA. Sleep/wake cycle in a group of hypertensive subjects: strength of the circadian and semicircadian components. In: Proceedings of the $7^{\text {th }}$ Latin American Symposium on Chronobiology; 2003 nov. 24-26; Tlaxcala. México: Sociedade Mexicana de Ciências Fisiológicas; 2003. v. 1, p. 1.

44. Tolonen H, Wolf H, Jakovljevic D, Kuulasmaa K. Review of surveys for risk factors of major chronic diseases and comparability of the results. In: European Health Risk Monitoring (EHRM) Project. [text on the Internet]. Oslo; 2002. [cited 2002 Oct 11] Available from: http://www.ktl.fi/ publications/ehrm/product/title.htm/

45. Arcuri EAM, Santos JLF, Rocha e Silva M. Pulse pressure as a function of cuff width. Braz J Med Biol Res. 1988;21(1):53-6.

46. Arcuri EAM, Santos JLF, Rocha e Silva M. Are obese people more likely to be diagnosed and treated than leaner subjects for hypertension? Controv Cardiol. 1989;1(1):24.

47. Arcuri EAM, Santos JLF, Rocha e Silva M. Is early diagnosis of hypertension a function of cuff width? J Hypertens. 1989;7(1):60-1.

48. Arcuri EAM. Manguito do esfigmomanômetro e diagnóstico de hipertensão arterial sistêmica. Arq Bras Cardiol. 1989;52(4):181-3.

49. Arcuri EAM, Martins E, Santos JLF. Correct versus standard cuff width: twenty years follow-up study. J Hypertens. 2004;22 Suppl 1:137.

50. Arcuri EAM, Araújo TL, Veiga EV, Oliveira SMJV, Lamas JLT, Santos JLF. The challenge of precise blood pressure measurement. In: Proceedings of the $16^{\text {th }}$ Scientific Meeting of the Interamerican Society of Hypertension; 2005 Apr 17-23; Cancún, Q.Roo. México; 2005. p. 185.

Agradecimentos: os autores agradecem a FAPESP, pelo apoio aos estudos pós-doutorais realizados no exterior e ao CNPq e FAPESP, pelas bolsas concedidas em diversas modalidades, propiciando a continuidade das investigações e formação de pesquisadores. 